\title{
Modeling Motivation for Alcohol in Humans using Traditional and Machine Learning Approaches
}

\author{
Erica N. Grodin ${ }^{1}$, Amanda K. Montoya ${ }^{1}$, Spencer Bujarski ${ }^{1}$, Lara A. Ray ${ }^{1,2}$ \\ ${ }^{1}$ University of California Los Angeles, Department of Psychology, Los Angeles, CA, USA \\ ${ }^{2}$ University of California Los Angeles, Department of Psychiatry and Biobehavioral Sciences, Los \\ Angeles, CA, USA
}

Corresponding Author: Lara A. Ray, Ph.D., Professor, University of California, Los Angeles, Psychology Department, 1285 Franz Hall, Box 951563, Los Angeles, CA 90095-1563; Phone: 310-794-5383; Fax: 310-206-5895; Email: lararay@ @sych.ucla.edu

Funding: This work was supported by grants from the National Institute on Alcohol Abuse and Alcoholism (NIAAA) to ENG (F32AA027699), to SB (F31AA022569), and to LAR (R21AA022752 and K24AA025704). None of the authors have conflicts of interest to disclose.

Acknowledgements: Support for the development of the Computer-Assisted Infusion System was provided by Sean O'Connor, Martin Plawecki, and Victor Vitvitskiy, Indiana Alcohol Research Center (P60 AA007611), Indiana University School of Medicine. 


\begin{abstract}
:
Given the significant cost of alcohol use disorder, identifying risk factors for alcohol seeking represents a research priority. Prominent addiction theories emphasize the role of motivation in the alcohol seeking process, which has largely been studied using preclinical models. In order to bridge the gap between preclinical and clinical studies, this study examined predictors of motivation for alcohol selfadministration using a novel paradigm. Heavy drinkers $(n=67)$ completed an alcohol infusion consisting of an alcohol challenge (target breath alcohol $=60 \mathrm{mg} \%$ ) and a progressive-ratio alcohol selfadministration paradigm (maximum breath alcohol 120mg\%; ratio requirements range $=20-3,139$ response). Growth curve modeling was used to predict breath alcohol trajectories during alcohol selfadministration. K-means clustering was used to identify motivated $(n=41)$ and unmotivated $(n=26)$ selfadministration trajectories. The data was analyzed using two approaches: a theory-driven test of a-priori predictors and a data-driven, machine learning model. In both approaches, steeper delay discounting, indicating a preference for smaller, sooner rewards, predicted motivated alcohol seeking. The data-driven approach further identified phasic alcohol craving as a predictor of motivated alcohol self-administration. Additional application of this model to AUD translational science and treatment development appear warranted.
\end{abstract}

Key words: motivation, alcohol seeking, machine learning, delay discounting, progressive ratio 
Running Head: Human Alcohol Motivation Model

\section{Introduction}

Alcohol use disorder (AUD) is a chronic relapsing condition, defined by consumption that continues despite significant physical, social, economic, and legal consequences. AUD is a significant public health concern; in the United States, over 15 million adults met criteria for an AUD and an estimated 623,000 adolescents ages 12-17 had an AUD ${ }^{1}$. Moreover, almost 27\% of adults reported engaging in a binge drinking episode in the past month, with 7\% reporting past month heavy drinking, defined as 5 or more binge drinking episodes in one month ${ }^{2}$. Given the significant cost of AUD to afflicted individuals and society at large, identifying risk factors for alcohol seeking in heavy drinkers and in individuals with AUD represents a research priority.

Prominent theories of addiction emphasize the critical role of motivation in the alcohol/drug seeking process, especially in early stages of the addiction cycle ${ }^{3,4}$. In preclinical animal models, motivation for alcohol is traditionally measured through behavioral assays that are often disparate from the human and clinical presentations. A common method for measuring alcohol's reinforcing value is the progressive ratio (PR) schedule, in which rodents must press a lever in increasing increments to earn an alcohol reward (i.e. a PR4 schedule would require 4, 8, 12, etc. lever presses for each subsequent alcohol reward). The maximum number of lever presses for the last complete reward trial is referred to as the breakpoint, which measures the amount of effort a subject (i.e., animal) is willing to perform to earn the reward. Providing validity for this approach, high-drinking rats have a higher breakpoint and consume more alcohol than low drinking rats in a PR design ${ }^{5}$. Importantly, PR breakpoint is sensitive to AUD pharmacotherapy effects, such that increasing doses of effective medication can decrease PR breakpoint, indicating that the medication decreases motivation for alcohol ${ }^{6}$. While much progress has been made in the preclinical domain using animal models of motivation for alcohol, translation of these models to humans has been limited.

Recently, the field has been moving towards translational research in AUD in which controlled laboratory models can be used to identify predictors of risk and vulnerability factors for AUD. In this 
Running Head: Human Alcohol Motivation Model

vein, a recent experimental study investigated if risk factors for AUD could predict rates of binge drinking during a controlled intravenous alcohol self-administration paradigm, albeit without a PR component ${ }^{7}$. Specifically, this study found that family history of alcoholism, male sex, and delay discounting impulsivity were associated with higher rates of binge drinking, defined as reaching a blood alcohol content of $80 \mathrm{mg} \%$. Further, individuals who met all three risk factors had higher rates of binge drinking during the self-administration paradigm compared to the lowest risk group, who did not have any AUD risk factors ${ }^{7}$, suggesting an additive effect. While this was an important study in validating alcohol self-administration through well-established AUD risk factors, models incorporating a PR schedule have arguably greater translational value.

In order to bridge the gap between preclinical and clinical studies of motivation for alcohol, the present study examines predictors of motivation for alcohol self-administration using a novel PR $\operatorname{paradigm}^{8}$. First, we used growth modeling and K-means clustering to identify motivated and unmotivated self-administration trajectories phenotyped in the laboratory. Then we used two analytic approaches to identify predictors of motivation for alcohol self-administration. First we conducted a theory-driven test of a-priori predictors, namely family history, sex, and impulsivity, selected to replicate and extend upon previous findings of vulnerability to alcohol bingeing ${ }^{7}$, using traditional inferential statistics (i.e., logistic regression). Second, we used a data-driven, machine learning approach, i.e. random forest model. Machine learning models prioritize the ability to predict future outcomes over creating perfectly fitting models for the data at hand ${ }^{9}$. This results in models which are more generalizable to future observations, which fits well with our goal of identifying predictors of motivation for alcoholseeking in clinical samples. Together, this approach combines novel experimental methods (PR selfadministration in humans) with advanced quantitative methods (growth modeling, random forest modeling) to extend the literature on determinants of motivation for alcohol seeking in humans. The combination of theory testing and data-driven modeling provides a balanced approach to this critical scientific question. 


\section{Method}

\section{Participants}

This study was approved by the UCLA Institutional Review Board. Non-treatment-seeking heavy drinkers were recruited between April 2015 and August 2016 from the Los Angeles community through fliers and online advertisements. Initial eligibility screening was conducted via online and telephone surveys and was followed by an in-person screening session. After providing written informed consent, participants were breathalyzed, provided urine for toxicology screening, and completed a battery of selfreport questionnaires and interviews. All participants were required to have a breath alcohol content (BrAC) of 0mg\% and to test negative on a urine drug screen for all drugs of abuse (except cannabis). Female participants were required to test negative on a urine pregnancy test. A physical examination was performed to ensure medical eligibility to complete an intravenous alcohol administration. Participants were between 21 and 45 years of age, current heavy drinkers based on NIAAA recommendations (14+ drinks per week for men or 7+ drinks per week for women), non-treatment-seeking for AUD, not undergoing significant alcohol withdrawal symptoms, and did not meet diagnostic criteria for any substance use disorder other than nicotine or alcohol.

\section{Alcohol Administration Procedure}

Alcohol administration was conducted at the UCLA Clinical and Translational Research Center (CTRC). Detailed methodology can be found in Bujarski et al., $2018^{8}$. In brief, upon arrival at the CTRC, height, weight, and vital signs were collected and the intravenous (IV) lines were placed by a nurse. Participants completed baseline questionnaire assessments. The alcohol infusion lasted 180 minutes and study staff were present throughout to monitor the infusion, breathalyze the participant, and administer questionnaires, but did not interact with the participant otherwise. To dissociate biobehavioral responses to alcohol from cue-induced responses and to enable precise control of $\mathrm{BrAC}$, alcohol was administered IV (6\% ethanol v/v in saline) using the Computerized Alcohol Infusion System (CAIS ${ }^{10-12}$ ). During the 
Running Head: Human Alcohol Motivation Model

alcohol challenge, participants were administered alcohol designed to reach target BrACs of 20, 40, and $60 \mathrm{mg} \%$, each over 15 minutes. BrACs were clamped at each target level while participants completed questionnaires ( $\sim 5$ minutes). Immediately after reaching $60 \mathrm{mg} \%$, participants began the progressive ratio self-administration paradigm. Participants could exert effort (by pressing an electronic button) to obtain additional "drinks" through the CAIS system, according to a log-linear progressive ratio schedule. Ratio requirements ranged from 20 responses ( $1^{\text {st }}$ completion) to 3139 responses $\left(20^{\text {th }}\right.$ completion). Each “drink" increased BrAC by $7.5 \mathrm{mg} \%$ over $2.5 \mathrm{~min}$, followed by a descent of $-1 \mathrm{mg} \% / \mathrm{min}^{11}$. There was a $120 \mathrm{mg} \%$ BrAC safety limit, such that if an infusion of alcohol was predicted (by the CAIS algorithm) to increase BrAC beyond this limit, the response button would be temporarily inactivated. Except for the first "drink" (i.e., infusion self-administration designed as a "sample" drink), participants were given no instructions for the self-administration paradigm other than they could choose to self-administered by pressing the button at the required effort levels. After $180 \mathrm{~min}$, the infusion ended and participants were monitored and waited for discharge. To ensure all participants were safe to discharge, and to disincentivize low-levels of self-administration, all participants were informed that they would remain at the CTRC for at least 4 additional hours following alcohol self-administration regardless of their selfadministration profile.

\section{Measures}

AUD Severity Measures: The Structured Clinical Interview for DSM-5 (SCID; adapted from First et al., 2015 ${ }^{13}$ ) assessed for lifetime and current AUD. The Clinical Institute Withdrawal Assessment for Alcohol (CIWA-Ar) assessed for alcohol withdrawal symptoms ${ }^{14}$. A 30-day Timeline Followback (TLFB) assessed drinking quantity and frequency over the past month ${ }^{15}$. Participants also completed the Alcohol Dependence Scale $\left(\mathrm{ADS}^{16}\right.$ ), the Alcohol Use Disorders Identification Test (AUDIT ${ }^{17}$ ), the Drinkers Inventory of Consequences (DrINC-2 $\mathrm{r}^{18}$ ), the Penn Alcohol Craving Scale $\left(\mathrm{PACS}^{19}\right.$ ), the Obsessive Compulsive Drinking Scale $\left(\mathrm{OCDS}^{20}\right)$, the Drinking Motives Questionnaire - Revised ${ }^{21}$, the UCLA Reward, Relief, Habit Drinking Scale (UCLA RRHDS ${ }^{22}$ ), and the Family Tree Questionnaire 
Running Head: Human Alcohol Motivation Model

$\left(\mathrm{FTQ}^{23}\right)$. A family history of AUD density score was calculated by dividing the number of relatives with reported alcohol problems by the total number of first- and second-degree relatives.

Individual Differences Measures: Cigarette and marijuana use quantity and frequency over the past month were assessed using the TLFB. The Fagerstrom Test for Nicotine Dependence assessed for nicotine dependence $\left(\mathrm{FTND}^{24}\right)$. Cannabis problems were assessed using the Cannabis Use Disorders Identification Test - Revised (CUDIT-R ${ }^{25}$ ). Depressive symptomatology was assessed via the Beck Depression Inventory - II (BDI-II $\left.{ }^{26}\right)$. Anxiety symptomatology was assessed using the State-Trait Anxiety Inventory $\left(\mathrm{STAI}^{27}\right)$. Delay discounting impulsivity was measured using the monetary choice questionnaire $\left(\mathrm{MCQ}^{28}\right)$. The MCQ was scored to calculate $k$, a constant that reflects the rate of discounting; a higher value of $k$ indicates the tendency to prefer smaller, sooner rewards over larger, delayed rewards. As $k$ has a strong positive skew, the data was normalized using logarithmic transformation.

Subjective Response to Alcohol (SR) Measures: Participants completed SR assessments at baseline, 20, 40, and $60 \mathrm{mg} \%$ time points during the alcohol challenge (and prior to progressive ratio selfadministration period). Based on a previous factor analysis, SR was assessed along four dimensions: stimulation/hedonia (stimulation), negative affect, sedation/motor intoxication (sedation), and craving ${ }^{29}$. The factor analysis used to derive these four dimensions of SR has been previously described ${ }^{8}$.

\section{Data Analysis}

First, we fit a growth curve model predicting BrAC averaged across 5 minute windows, allowing for a fourth degree polynomial effect of time, a linear effect of alcohol severity, and for the effect of alcohol use severity to interact with the effects of time (see Burjarski et al., $2018^{8}$ for model selection process). All trial specific predictors were allowed to have random effects (i.e., all time predictors were allowed to have random effects, but alcohol use severity and interactions which alcohol use severity were all fixed effects). The random effects for each participant were extracted and submitted to a K-means 
Running Head: Human Alcohol Motivation Model

cluster analysis initially testing both two and three clusters. By extracting the random effects, and submitted them to a cluster analysis, we are able to identify unique trajectories of change during the selfadministration task. All analyses were conducted in R.

Two analytic approaches were taken to predict motivation for alcohol self-administration cluster membership. First, a "traditional approach" consisting of logistic regression analysis was conducted using three $a$-priori variables based on earlier work ${ }^{7}$. The predictive variables included in this analysis were: delay discounting $(\log (k))$, family history of AUD (positive or negative), and gender. Second, a data driven approach consisting of a random forest model was run to identify clinical predictors of whether individuals would fall into the motivated or unmotivated alcohol self-administration trajectories. For the random forest the data was split into two parts, training data $(n=33)$ and testing data $(n=34)$. Typical recommendations for splitting training and testing data suggest $2 / 3$ to training and $1 / 3$ to testing; however, for smaller sample sizes a half split tends to result in a better estimate of prediction accuracy ${ }^{30}$. The training data is used to create the model and the testing data is used to evaluate the model's ability to predict observations not used to create the model (out-of-sample prediction). A random forest is an aggregate of many classification trees. In this model, we created 500 classification trees, each tree is created from a bootstrap sample of the training data, for each node of each tree 6 variables were randomly selected from the 32 candidate predictors, and the best candidate is selected and the data is split based on the predictor. By limiting the number of candidate predictors for each tree, random forests are able to estimate the importance of variables equally, even those variables that are highly correlated with other predictors $^{31}$. The recommended number of variables used for each tree is the square root of the number of variables (in this case 5.66), so 6 variables were used. Predictions for each observation in the test dataset are generated based on the majority vote from all 500 trees. Variable importance rating and partial dependence plots were used to determine the most important variables in the model and the marginal effect they have on the outcome. The variables included as candidate predictors were: age, gender, AUD severity, current AUD diagnosis, total number of drinking days (30 days), drinks per drinking day (30 days), AUDIT total score, ADS total score, drinking motive dimensions (enhancement, social, 
Running Head: Human Alcohol Motivation Model

conformity, and coping), dichotomized family history of alcoholism (positive or negative), PACS score, RRDS scores for reward, relief, and habit, BDI-II score, STAI score, cigarette smoking days (30 days smokers only), cigarettes per smoking day (30 days - smokers only), cannabis use days (30 days cannabis users only), CUDIT score (cannabis users only), log transformed delayed discounting $(\log (k))$, and subjective response measures from the alcohol challenge (peak ratings and time of peak for stimulation, sedation, negative affect, and craving). The broad set of variables selected was meant to capture plausible predictors while capitalizing on a robust data analytic model that can handle a multitude of predictor variables in spite of a moderate sample size.

\section{Results}

\section{K-Means Cluster Analysis}

For the cluster analysis, a silhouette plot indicated that 2 clusters were more optimal than 3 . One cluster was characterized by a motivated phenotype $(n=41)$, such that individuals in this group exhibited effort to continue receiving alcohol throughout the self-administration progressive ratio session. The other cluster was characterized by an unmotivated phenotype $(n=26)$, such that individuals in this group did not exert increasing amounts of effort to earn alcohol infusions throughout the session and therefore had decreasing BrAC during the self-administration paradigm (see Figure 1). The average breakpoint for individuals in the motivated cluster was $871.61 \pm 368.53$ (maximum breakpoint $=1,1775$, resulting in a BrAC of $117.93 \mathrm{mg} \%)$. The average breakpoint for individuals in the unmotivated cluster was 186.08 \pm 255.07 (maximum breakpoints $=1,019$, resulting in a $\mathrm{BrAC}$ of $99.18 \mathrm{mg} \%$ ). There were no significant differences in demographic characteristics or in alcohol use measures between the two selfadministration groups (see Table 1). 


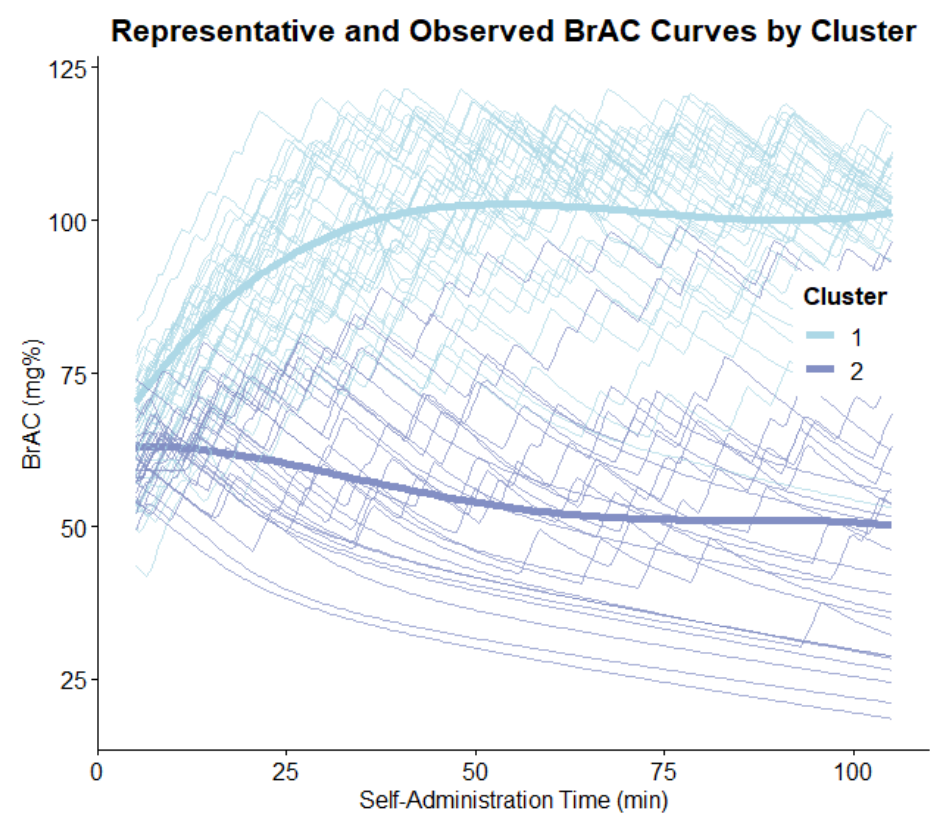

Figure 1. BrAC over time during the selfadministration task. Lines with smaller weight are observed BrAC curves from each individual, colored based on their assigned cluster where light blue indicates the motivated cluster (Cluster 1) and dark blue indicates unmotivated cluster (Cluster 2). The two heavily weighted lines indicate the representative curve for each cluster, based on parameters from the cluster analysis. Model

was fit using averages over 5 minute periods, resulting in slight differences between groups at onset due to group differences in behavior within the first 5 minutes.

\section{Table 1. Participant Characteristics}

\begin{tabular}{|c|c|c|c|c|}
\hline & Motivated (n=41) & Unmotivated $(\mathrm{n}=26)$ & Statistic & p-value \\
\hline Age & $29.88 \pm 6.53$ & $27.85 \pm 6.68$ & $\mathrm{t}=1.23$ & 0.22 \\
\hline Gender $(\mathrm{M} / \mathrm{F})$ & $23 / 18$ & $13 / 13$ & $X^{2}=0.24$ & 0.63 \\
\hline Ethnicity & & & $X^{2}=0.62$ & 0.43 \\
\hline White, Non-Hispanic & 36 & 21 & & \\
\hline White, Hispanic & 5 & 5 & & \\
\hline Education & & & $X^{2}=2.52$ & 0.77 \\
\hline$<12$ years & 1 & 0 & & \\
\hline 12 years (Highschool) & 10 & 9 & & \\
\hline 14 years (Associates) & 8 & 3 & & \\
\hline 16 years (College) & 16 & 11 & & \\
\hline 18 years (Masters) & 5 & 3 & & \\
\hline $20+$ years $(\mathrm{MD}, \mathrm{JD})$ & 1 & 0 & & \\
\hline Income & & & $X^{2}=5.82$ & 0.67 \\
\hline$<\$ 15,000$ & 8 & 7 & & \\
\hline$\$ 15,000-\$ 29,999$ & 13 & 6 & & \\
\hline$\$ 30,000-\$ 44,999$ & 5 & 4 & & \\
\hline$\$ 45,000-\$ 59,999$ & 7 & 3 & & \\
\hline$\$ 60,000-\$ 74,999$ & 2 & 3 & & \\
\hline$\$ 75,000-\$ 89,999$ & 1 & 1 & & \\
\hline$\$ 90,000-\$ 104,999$ & 1 & 2 & & \\
\hline$\$ 105,000-\$ 119,999$ & 1 & 0 & & \\
\hline$>\$ 120,000$ & 3 & 0 & & \\
\hline
\end{tabular}


Running Head: Human Alcohol Motivation Model

\begin{tabular}{lllll} 
Alcohol Use & & & \\
AUD Current Symptom Count & $2.41 \pm 1.82$ & $2.46 \pm 2.50$ & $\mathrm{t}=0.09$ & 0.93 \\
CIWA-Ar & $1.00 \pm 1.16$ & $1.08 \pm 1.67$ & $\mathrm{t}=0.22$ & 0.83 \\
Total Drinks (30 days) & $93.20 \pm 61.61$ & $96.48 \pm 48.56$ & $\mathrm{t}=0.23$ & 0.82 \\
Drinks per drinking day (30 days) & $5.39 \pm 2.64$ & $5.16 \pm 2.48$ & $\mathrm{t}=0.37$ & 0.72 \\
ADS & $11.80 \pm 5.38$ & $10.04 \pm 5.54$ & $\mathrm{t}=1.30$ & 0.20 \\
AUDIT & $13.39 \pm 5.55$ & $13.50 \pm 6.39$ & $\mathrm{t}=0.07$ & 0.94 \\
OCDS & $8.39 \pm 5.02$ & $9.00 \pm 4.65$ & $\mathrm{t}=0.50$ & 0.62 \\
PACS & $9.68 \pm 5.81$ & $9.85 \pm 5.92$ & $\mathrm{t}=0.11$ & 0.91 \\
Family History (Negative/Positive) & $20 / 17$ & $11 / 13$ & $\mathrm{X}=0.39$ & 0.53 \\
Family History Density & $0.20 \pm 0.27$ & $0.20 \pm 0.26$ & $\mathrm{t}=0.02$ & 0.99 \\
\hline
\end{tabular}

\section{Logistic Regression}

In the logistic regression analysis in which delay discounting $(\log (k))$, family history of AUD, and gender were simultaneously entered in the model, only delay discounting $(\log (k))$ was a significant predictor of cluster group membership $\left(B=-0.54, S E=0.23, \chi^{2}=5.50, p=0.02\right)$. Neither gender nor presence or absence of family history significantly predicted self-administration phenotype (gender: $B=-$ $0.18, S E=0.59, \chi^{2}=0.09, p=0.76$; family history: $\left.B=0.21, S E=0.56, \chi^{2}=0.14, p=0.71\right)$. Results were such that more impulsive individuals, characterized by delay discounting response profiles, were more likely to fall in the "motivated group."

\section{Random Forest Models}

Using the machine learning approach, two clinical variables emerged as the most reliable predictors of cluster membership: craving for alcohol during the alcohol challenge and delay discounting $((\log (k))$; see Figure 2 for a representative random forest model). 


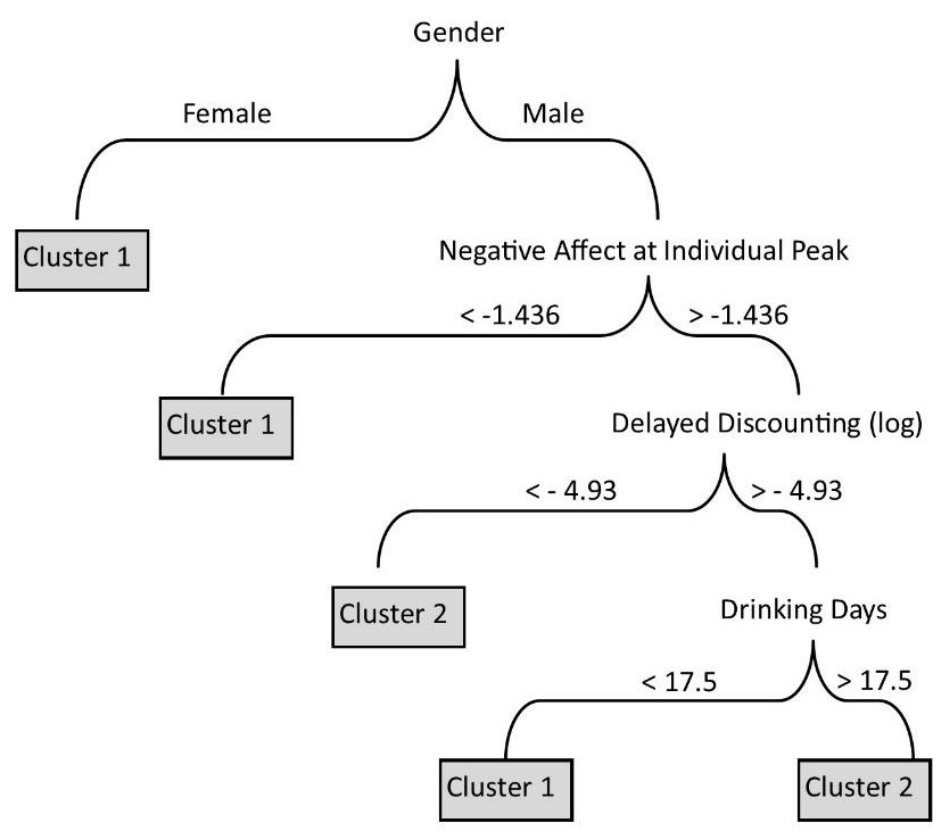

Figure 2. One tree randomly drawn from set of 500 trees fit as part of the random forest. The first variable splits by gender, where women are classified in Cluster 1 (motivated). Men are split by negative affect at individual peak where men lower than -1.436 are in Cluster 1 (motivated) and those higher than -1.436 are then split by delayed discounting. Men higher than 1.436 on negative affect at individual peak who are lower than -4.93 on delayed discounting are classified in Cluster 2 (unmotivated). Those remaining who are higher than -4.93 on delayed discounting are then split by Drinking Days, where individuals will fewer than 17.5 drinking days are assigned Cluster 1 (motivated) and those with more than 17.5 drinking days are assigned Cluster 2 (unmotivated).

Alcohol craving during the alcohol challenge was predictive of the self-administration profile, such that individuals who reported greater alcohol craving during the alcohol challenge phase were more likely to be in the motivated alcohol self-administration group. Similarly, delay discounting was predictive of the self-administration profile, such that individuals who were more impulsive, (i.e. discounted future rewards at a steeper rate), were more likely to be in the motivated self-administration group. As shown in Figure 3, partial dependence plots depict the marginal relationship between a predictor and the relative probability of being in the motivated vs. the unmotivated cluster. These plots were used to inform our interpretation of directionality as well as the linear/non-linear nature of the results. In order to evaluate the performance of the model, the model fit with the training data was then used to predict the observations of the testing data to estimate ability to predict out-of-sample observations. Out of sample results were acceptable, where in the sample of 34 participants, 20 were correctly classified in the motivated cluster 
and 4 were correctly classified in the unmotivated cluster (70.6\%). Whereas 1 participant was incorrectly classified in the motivated cluster and 9 were incorrectly classified in the unmotivated cluster (29.4\%). This classification performs significantly better than chance $\left(\chi^{2}=4.33, p=0.037\right)$. Notably, the error in classification is not symmetric, and the model seems to do a good job of identifying participants in the motivated cluster, but is less reliable at classifying participants in the unmotivated cluster.
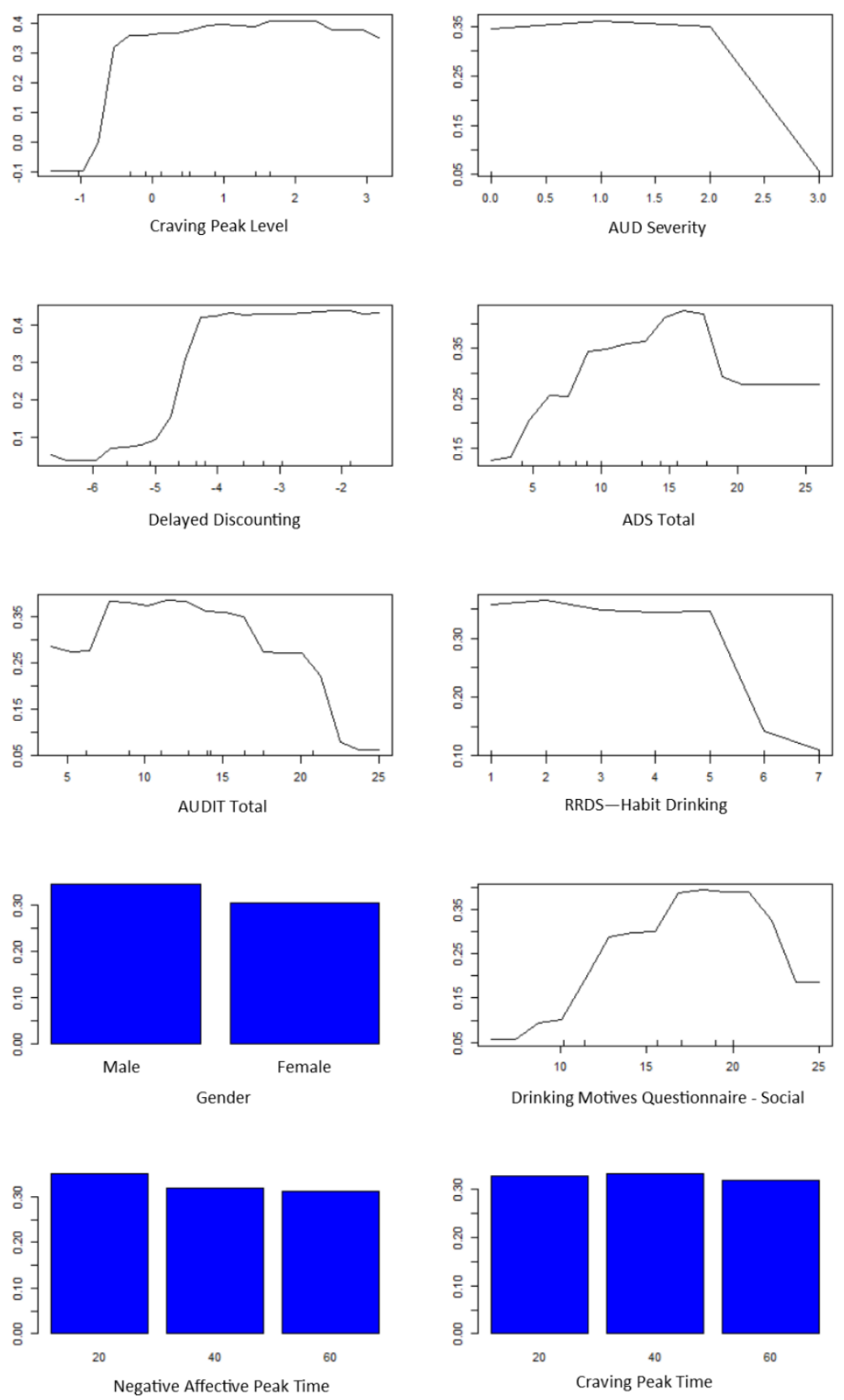

Figure 3. Partial dependence plots show the marginal relationship between a predictor (along the $\mathrm{X}$ axis) and the relative probability of being in Cluster 1 vs Cluster 2 on the Y-axis. In particular, positive scores indicate higher likelihood of belonging to Cluster 1 (motivated) and negative scores indicate higher likelihood of belonging to Cluster 2 (unmotivated). For example, for individual's whose peak craving is relatively low (around -1) they are more likely to be in Cluster 2 (unmotivated) but with higher peak craving levels, individuals are more likely to be in Cluster 1 (motivated). However, this relationship does not seem to be

linear, rather there is a particular threshold which defines the differences between the groups. 


\section{Discussion}

This study sought to bridge the gap between preclinical and clinical studies of motivation for alcohol seeking by examining predictors of alcohol self-administration phenotypes. First, we identified two clusters of alcohol self-administration phenotypes based on observed self-administration in the laboratory: motivated and unmotivated. Next, using theory-driven, a-priori predictors, we found that delay discounting predicted cluster membership in a logistic regression model. Specifically, individuals with steeper delay discounting curves (i.e. more impulsive) were more likely to be classified as motivated during alcohol self-administration. Finally, in the random forest models the most reliable predictors of alcohol self-administration phenotype were delay discounting impulsivity and craving for alcohol during an alcohol challenge.

Notably, both the theory-driven and the data-driven methods identified delay discounting impulsivity as a predictor of alcohol self-administration phenotype. In both methods, individuals who tended to choose smaller, sooner rewards over larger, later rewards were more likely to be classified as motivated for alcohol seeking. Delay discounting was previously observed as a predictor of laboratory alcohol consumption and was predictive of binge alcohol consumption ${ }^{7,32}$. Delay discounting impulsivity has been identified as a candidate behavioral marker of addiction, as it is increased in individuals with addictive disorders and can identify individuals at risk for addictive disorders ${ }^{33}$. Intriguingly, delay discounting was also found to be the most robust classifier in a classification tree analysis to distinguish alcohol and stimulant users from controls ${ }^{34}$. These results fit well with the recent reinforcer pathology theory, which highlights the interaction between excessive preference for the drug over other rewards and excessive preference for immediate rewards ${ }^{35}$. In the present study, individuals who were motivated to seek alcohol choose to work for this preferred reward over the more relaxing choice of not engaging in effort and showed a preference for smaller, sooner rewards. The reinforcer pathology theory predicts that individuals who demonstrate both a high demand for their preferred drug as well as a high preference for immediate rewards are at the highest risk to initiate substance use. 
Running Head: Human Alcohol Motivation Model

Importantly, delay discounting may be targeted and modified through behavioral interventions. For example contingency management for cigarette smoking was found to decrease delay reward discounting ${ }^{36}$. Furthermore, novel interventions such as episodic future thinking and altering temporal attention were also found to dampen delay discounting ${ }^{37-39}$. In individuals with AUD, training in episodic future thinking, where individuals are required to vividly experience a realistic event in the future, increased the value of future monetary rewards and decreased hypothetical initial consumption of alcoholic drinks, particularly in individuals with lower AUDIT scores ${ }^{40}$. In the current study, all participants were non-treatment-seeking and the majority of participants had comparable AUDIT scores, indicating that this training may be particularly valuable for improving delay discounting and future drinking outcomes in individuals with the motivated alcohol-self administration phenotype.

The data-driven, machine learning, approach also identified alcohol craving during the alcohol challenge as a predictor of alcohol self-administration. Specifically, individuals who reported higher peak ratings of craving for alcohol during the alcohol challenge were more likely to be classified as motivated for alcohol seeking during the progressive ratio self-administration session. This result extends our previous work which identified alcohol craving as a more proximal predictor of alcohol selfadministration than other subjective responses, including stimulation and sedation ${ }^{41}$. In the laboratory, naltrexone, an FDA approved treatment for AUD, reduces alcohol self-administration and alcohol craving $^{42,43}$, indicating that this behavioral marker of alcohol seeking may also be targeted through pharmacotherapy. In essence, the convergence of findings across two distinct and robust analytic methods along with validation within the broader literature suggests that this translational paradigm may be useful in advancing AUD research.

Machine learning methods are playing an increasingly important role in psychological science, in particular, within the area of clinical psychology. Research focused on clinical outcomes and prevention cannot just prioritize creating models which fit well with sample data, but also models which do a good job of predicting future observations. Machine learning models tend to prioritize the ability to predict 
future outcomes over creating perfectly fitting models for the data at hand. This results in models which are more generalizable to future observations. For example, Leach et al., $2016^{44}$ used a similar approach to random forests, decision trees, to identify important environmental characteristics which contribute to cardiovascular disease in African American women. This example brings about another advantage of machine learning approaches, which is that many are well suited for selecting among a large set of candidate predictors ${ }^{45}$. In the current study, due to small sample size, the number of predictors which were reasonable to use in the logistic regression was very limited; however, with the random forest analysis we were able to include 32 predictors. This is because random forests choose which predictors to include in the model, whereas linear and logistic regression approaches always include all predictors in the model, taking up available degrees of freedom. However, with these models comes a particular limitation that will be important to acknowledge in clinical frameworks: a lack of hypothesis testing or thresholds to determine which predictors are "significant" and which are not. In this study, we focused on the top two predictors, as these were consistent across multiple runs of the model and showed the greatest variable importance. However, even in models where no predictors predict above chance, random forest will always rank order variables based on importance. By combining traditional and machine learning approaches, researchers can benefit from the exploratory nature of the machine learning models, while still estimating inferential tests using traditional models. Coughlin and colleagues used machine learning approaches to predict smoking cessation status following psychosocial treatment ${ }^{46}$. Notably, delay discounting impulsivity was the best predictor of treatment response, further highlighting the key role of delay discounting in addiction processes. As precision medicine becomes increasingly common in both clinical psychology settings and other medical settings more broadly, machine learning approaches can be utilized to comb through large sets of candidate moderators. This means that based on individual's characteristics we can make precise predictions about what the best possible treatment would be for each individual. Machine learning approaches have only recently been found within psychological research ${ }^{47}$; however, their potential value to clinical research is very high, and our study demonstrates one of the potential advantages of using machine learning for improving our understanding of clinical outcomes. 
Running Head: Human Alcohol Motivation Model

The present study should be interpreted in light of its strengths and limitations. Strengths include the use of traditional and data-driven approaches and the use of a novel paradigm for alcohol selfadministration in humans. One limitation is that the self-administration paradigm occurred directly following the alcohol challenge, and thus all participants in the study were already experiencing the effects of alcohol prior to beginning the alcohol progressive ratio task. It is unknown if the pre-selfadministration alcohol challenge altered performance on the progressive ratio task and future studies should explore this issue. Relatedly, due to safety concerns, BrAC in the present study was capped at $120 \mathrm{mg} \%$, which may have limited self-administration as individuals were prevented from pressing for alcohol when they would go over the safety limit. Moreover, this study did not include a placebo infusion, which limits our ability to make causal conclusions about motivation to seek alcohol in this paradigm. Additionally, this study was cross-sectional in design and is therefore unable to determine if the alcohol self-administration phenotypes identified truly reflect risk factors for the development of AUD or alcoholrelated problems. Longitudinal studies are needed to confirm that these patterns are vulnerability factors. Further, this study employed an intravenous alcohol administration paradigm, which may have limited ecological validity for real-world alcohol consumption. However, a recent prospective study found that IV alcohol self-administration was predictive of real-world heavy drinking ${ }^{48}$. Finally, this study had a relatively small sample size, which may have limited our ability to detect group differences in the a priori variables in the traditional statistical analyses.

In conclusion, the present study leveraged a translational experimental paradigm of progressive ratio self-administration in combination with theory- and data-driven methods and found that both delayed reward discounting and alcohol craving during an alcohol challenge, predicted motivation towards alcohol self-administration. Based on this robust combination of a translational paradigm for alcohol-seeking with traditional and novel analytic approaches, it appears that additional application of this model to AUD translational science and treatment development are warranted. 
Running Head: Human Alcohol Motivation Model

Contributions: ENG and LAR developed the study concept. Testing and data collection were performed by SB. ENG, AKM, and SB performed the data analysis. ENG and LAR conducted the data interpretation. ENG drafted the paper and all authors provided critical revisions. All authors approved the final version of the paper for submission.

Data Availability: The data that support the findings of this study are openly available in Github at https://github.com/sbujarski/Bujarski_et_al_2018_NPP. 


\section{References}

1. Grant BF, Goldstein RB, Saha TD, et al. Epidemiology of DSM-5 Alcohol Use Disorder Results From the National Epidemiologic Survey on Alcohol and Related Conditions III. Jama Psychiat. 2015;72(8):757-766.

2. Administration SAaMHS. 2015 National Survey on Drug Use and Health (NSDUH). Table 2.46 B-Alcohol Use, Binge Alcohol Use, and Heavy Alcohol Use in Past Month among Persons Aged 12 or Older, by Demographic Characteristics: Percentages, 2014 and 2015. In:2015.

3. Koob GF, Volkow ND. Neurobiology of addiction: a neurocircuitry analysis. Lancet Psychiatry. 2016;3(8):760-773.

4. Olney JJ, Warlow SM, Naffziger EE, Berridge KC. Current perspectives on incentive salience and applications to clinical disorders. Curr Opin Behav Sci. 2018;22:59-69.

5. Spoelder M, Hesseling P, Baars AM, et al. Individual Variation in Alcohol Intake Predicts Reinforcement, Motivation, and Compulsive Alcohol Use in Rats. Alcoholism: Clinical and Experimental Research. 2015;39(12):2427-2437.

6. Augier E, Dulman RS, Damadzic R, Pilling A, Hamilton JP, Heilig M. The GABAB Positive Allosteric Modulator ADX71441 Attenuates Alcohol Self-Administration and Relapse to Alcohol Seeking in Rats. Neuropsychopharmacology. 2017;42(9):1789-1799.

7. Gowin JL, Sloan ME, Stangl BL, Vatsalya V, Ramchandani VA. Vulnerability for alcohol use disorder and rate of alcohol consumption. American Journal of Psychiatry. 2017;174(11):10941101.

8. Bujarski S, Jentsch JD, Roche DJO, Ramchandani VA, Miotto K, Ray LA. Differences in the subjective and motivational properties of alcohol across alcohol use severity: application of a novel translational human laboratory paradigm. Neuropsychopharmacology. 2018;43(9):18911899.

9. Hastie T, Tibshirani R, Friedman J. The Elements of Statistical Learning: Data Mining, Inference, and Prediction. New York, NY: Springer; 2001.

10. Plawecki MH, Han JJ, Doerschuk PC, Ramchandani VA, O'Connor SJ. Physiologically based pharmacokinetic (PBPK) models for ethanol. IEEE Trans Biomed Eng. 2008;55(12):2691-2700.

11. Zimmermann US, Mick I, Vitvitskyi V, Plawecki MH, Mann KF, O'Connor S. Development and pilot validation of computer-assisted self-infusion of ethanol (CASE): a new method to study alcohol self-administration in humans. Alcohol Clin Exp Res. 2008;32(7):1321-1328.

12. Zimmermann US, O'Connor S, Ramchandani VA. Modeling alcohol self-administration in the human laboratory. Curr Top Behav Neurosci. 2013;13:315-353.

13. First M, Williams J, Karg R, RL S. Structured Clinical Interview for the DSM-5. Arlington, VA: American Psychiatric Association; 2015.

14. Sullivan JT, Sykora K, Schneiderman J, Naranjo CA, Sellers EM. Assessment of alcohol withdrawal: the revised clinical institute withdrawal assessment for alcohol scale (CIWA-Ar). Addiction. 1989;84(11):1353-1357.

15. Sobell LC, Sobell MB, Leo GI, Cancilla A. Reliability of a timeline method: Assessing normal drinkers' reports of recent drinking and a comparative evaluation across several populations. British journal of addiction. 1988;83(4):393-402.

16. Skinner HA, Allen BA. Alcohol dependence syndrome: measurement and validation. Journal of abnormal psychology. 1982;91(3):199.

17. Allen JP, Litten RZ, Fertig JB, Babor T. A review of research on the Alcohol Use Disorders Identification Test (AUDIT). Alcoholism: clinical and experimental research. 1997;21(4):613619.

18. Miller W, Tonigan J, Longabaugh R. The Drinker Inventory of Consequences (DrInC): An instrument for assessing adverse consequences of alcohol abuse (Vol. 4). Rockville, MD: National Institute on Alcohol Abuse and Alcoholism. 1995. 
19. Flannery B, Volpicelli J, Pettinati H. Psychometric properties of the Penn alcohol craving scale. Alcoholism: Clinical and Experimental Research. 1999;23(8):1289-1295.

20. Anton RF. Obsessive-compulsive aspects of craving: development of the Obsessive Compulsive Drinking Scale. Addiction. 2000;95(8s2):211-217.

21. Cooper ML. Motivations for alcohol use among adolescents: Development and validation of a four-factor model. Psychological assessment. 1994;6(2):117.

22. Grodin EN, Bujarski S, Venegas A, et al. Reward, Relief and Habit Drinking: Initial Validation of a Brief Assessment Tool. Alcohol Alcohol. 2019.

23. Mann RE, Sobell LC, Sobell MB, Pavan D. Reliability of a family tree questionnaire for assessing family history of alcohol problems. Drug Alcohol Depend. 1985;15(1-2):61-67.

24. Heatherton TF, Kozlowski LT, Frecker RC, FAGERSTROM KO. The Fagerström test for nicotine dependence: a revision of the Fagerstrom Tolerance Questionnaire. British journal of addiction. 1991;86(9):1119-1127.

25. Adamson SJ, Kay-Lambkin FJ, Baker AL, et al. An improved brief measure of cannabis misuse: the Cannabis Use Disorders Identification Test-Revised (CUDIT-R). Drug Alcohol Depend. 2010;110(1-2):137-143.

26. Beck AT, Steer RA, Brown GK. Beck depression inventory-II. San Antonio. 1996;78(2):490-498.

27. Spielberger CD. State-Trait anxiety inventory. The Corsini encyclopedia of psychology. 2010:1-1.

28. Kirby KN, Petry NM, Bickel WK. Heroin addicts have higher discount rates for delayed rewards than non-drug-using controls. Journal of Experimental psychology: general. 1999;128(1):78.

29. Bujarski S, Hutchison KE, Roche DJ, Ray LA. Factor Structure of Subjective Responses to Alcohol in Light and Heavy Drinkers. Alcohol Clin Exp Res. 2015;39(7):1193-1202.

30. Dobbin KK, Simon RM. Optimally splitting cases for training and testing high dimensional classifiers. BMC Med Genomics. 2011;4:31.

31. Breiman L. Random forests. Mach Learn. 2001;45(1):5-32.

32. Stangl BL, Vatsalya V, Zametkin MR, et al. Exposure-Response Relationships during FreeAccess Intravenous Alcohol Self-Administration in Nondependent Drinkers: Influence of Alcohol Expectancies and Impulsivity. Int J Neuropsychoph. 2017;20(1).

33. Bickel WK, Koffarnus MN, Moody L, Wilson AG. The behavioral- and neuro-economic process of temporal discounting: A candidate behavioral marker of addiction. Neuropharmacology. 2014;76 Pt B:518-527.

34. Bickel WK, Moody LN, Eddy CR, Franck CT. Neurocognitive dysfunction in addiction: Testing hypotheses of diffuse versus selective phenotypic dysfunction with a classification-based approach. Exp Clin Psychopharmacol. 2017;25(4):322-332.

35. Bickel WK, Snider SE, Quisenberry AJ, Stein JS. Reinforcer Pathology: The Behavioral Economics of Abuse Liability Testing. Clin Pharmacol Ther. 2017;101(2):185-187.

36. Yi R, Johnson MW, Giordano LA, Landes RD, Badger GJ, Bickel WK. The Effects of Reduced Cigarette Smoking on Discounting Future Rewards: An Initial Evaluation. Psychol Rec. 2008;58(2):163-174.

37. Radu PT, Yi R, Bickel WK, Gross JJ, McClure SM. A Mechanism for Reducing Delay Discounting by Altering Temporal Attention. J Exp Anal Behav. 2011;96(3):363-385.

38. Sheffer CE, Mackillop J, Fernandez A, et al. Initial examination of priming tasks to decrease delay discounting. Behav Process. 2016;128:144-152.

39. Stein JS, Tegge AN, Turner JK, Bickel WK. Episodic future thinking reduces delay discounting and cigarette demand: an investigation of the good-subject effect. J Behav Med. 2018;41(2):269276.

40. Snider SE, LaConte SM, Bickel WK. Episodic Future Thinking: Expansion of the Temporal Window in Individuals with Alcohol Dependence. Alcoholism: Clinical and Experimental Research. 2016;40(7):1558-1566. 
41. Green R, Grodin E, Lim AC, et al. The Interplay Between Subjective Response to Alcohol, Craving, and Alcohol Self-Administration in the Human Laboratory. Alcoholism: Clinical and Experimental Research. 2019;43(5):907-915.

42. Hendershot CS, Wardell JD, Samokhvalov AV, Rehm J. Effects of naltrexone on alcohol selfadministration and craving: meta-analysis of human laboratory studies. Addiction Biology. 2017;22(6):1515-1527.

43. Ray LA, Green R, Roche DJO, Magill M, Bujarski S. Naltrexone effects on subjective responses to alcohol in the human laboratory: A systematic review and meta-analysis. Addiction Biology.0(0).

44. Leach HJ, O'Connor DP, Simpson RJ, Rifai HS, Mama SK, Lee RE. An exploratory decision tree analysis to predict cardiovascular disease risk in African American women. Health Psychol. 2016;35(4):397-402.

45. McNeish DM. Using Lasso for Predictor Selection and to Assuage Overfitting: A Method Long Overlooked in Behavioral Sciences. Multivar Behav Res. 2015;50(5).

46. Coughlin LN, Tegge AN, Sheffer CE, Bickel WK. A machine-learning approach to predicting smoking cessation treatment outcomes. Nicotine Tob Res. 2018.

47. Yarkoni T, Westfall J. Choosing Prediction Over Explanation in Psychology: Lessons From Machine Learning. Perspect Psychol Sci. 2017;12(6):1100-1122.

48. Hendershot CS, Wardell JD, McPhee MD, Ramchandani VA. A prospective study of genetic factors, human laboratory phenotypes, and heavy drinking in late adolescence. Addiction Biology. 2017;22(5):1343-1354. 